\title{
Bacterial isolates and their antibiotic sensitivity pattern of surgical site infections among the surgical ward patients of Asella Referral and Teaching Hospital
}

\author{
Teshome Gebissa ${ }^{1}$, Bonge Bude ${ }^{2}$, Mohd Yasir ${ }^{1}$, Shimelis Mekit ${ }^{1}$ and K. M. Noorulla ${ }^{1 *}$ (D)
}

\begin{abstract}
Background: Surgical site infection (SSI) is the most typical problem for patients who undergo operative procedures. It remains a typical and widespread problem causing morbidity and mortality, partly related to a rise in infections due to antimicrobial-resistant bacterial pathogens. The study was purposed to evaluate the bacterial isolates and their drug susceptibility patterns in patients with postoperative surgical site infection.

Results: The rate of postoperative surgical site infections was studied at Asella Referral and Teaching Hospital and found to be $23.3 \%$. One hundred fifty specimens of pus and surface swabs were collected from the surgical siteinfected patients over the period of March 2016 to May 2017, and from that, a total of 147 bacterial pathogens were recovered. The predominant organisms associated with postoperative surgical site infections were Klebsiella species 38 (26\%, n=147), Escherichia coli 31 (21\%, n=147), Staphylococcus aureus 25 (17\%, $n=147)$, and Pseudomonas aeruginosa $18(12 \%, n=147)$.

Conclusion: Higher number of bacterial isolates were recovered. The predominant isolates were Klebsiella species, Escherichia coli, Staphylococcus aureus, and Pseudomonas aeruginosa. The majority of gram-negative bacterial species which were isolated were found to be resistant to the commonly prescribed antimicrobial agents in the study setting. Thus, to achieve effective therapy for wound infections and to reduce/stop the appearance of multidrugresistant (MDR) pathogens, continuous monitoring is essential with the fair use of antimicrobial agents.
\end{abstract}

Keywords: Bacterial pathogen, Postoperative surgical site infection (SSI), Antibiotic sensitivity

\section{Background}

The hospital setting is a possible source of microbial infections as it lodges a large number of susceptible individuals and patients with various pathogenic microorganisms. In the hospital setting, the augmented prevalence of pathogenic microorganisms is correlated with a concurrent increase in different forms of nosocomial infections [1].

Surgical site infection (SSI) is characterized as an infection at the surgical site within 30 days after the

\footnotetext{
*Correspondence: nooruas@gmail.com

'Department of Pharmacy, College of Health Sciences, Arsi University, Asella, Oromia, Ethiopia

Full list of author information is available at the end of the article
}

surgery (or within 1 year if the implant is left in place), affecting either the incision or the deep tissue. These infections can involve apparent or deep tissue infections, organ or cavity infections [2]. Surgical site infections are the severe issues in the hospital population problems for patients undergoing surgeries, with the third most commonly identified hospital-acquired infection [2]. Intensified illness, extended hospitalization, death, and substantial financial burden for medical care are associated with postoperative surgical site infections $[3,4]$.

SSI prevention strategies have been highly developed, including enhanced ventilation of the operating theater, sterilization techniques, medical and surgical procedures, 
and the availability of prophylactic antibiotics. However, due to the proliferation of resistant pathogens, these SSIs are significant sources of morbidity and mortality [1]. The improper use of surgical prophylactic antibiotics leads partially to SSIs [3].

Antimicrobial resistance is a complex factor in controlling infections. It can increase complications and costs associated with procedures and treatment. An infected site complicates the postoperative course and results in a prolonged hospital stay and delayed recovery [5]. Advances in the treatment of diseases have contributed to a considerable rise in various surgical procedures. In response to these advancements, the severity of SSIs, antimicrobial prophylaxis, and the proliferation of antibiotic-resistant pathogens are expected to increase [5].

Certain life-saving medical procedures, like appendectomies and cesarean operations, are related to a high chance of infections and even mortality in underdeveloped countries, especially those with limited medical resources. Consequently, infections associated with the postoperative procedure usually increase the patient's hospital stay by an average of 7 to 10 days, and in some instances, it may cause the death of the patient $[6,7]$.

For a long time, postoperative infections associated with surgery have been an established crisis. Researchers identified the various types of pathogens from the surgical wound in patients related to Ethiopia. These pathogens are Staphylococcus aureus and certain species associated with Klebsiella, Enterobacter, Proteus, Pseudomonas, Streptococcus, and coagulase-negative Staphylococcus (CoNS) [8, 9]. The rate of hospital-acquired infection is distinctly elevated in many developing countries like Ethiopia, particularly for mostly preventable infections (e.g., those following surgical operations). For example, in Mekelle Hospital, Ethiopia, the incidence of postoperative surgical site infection was $44.1 \%$ of the patients with hospital-acquired infection [10].

Due to antimicrobial-resistant infections and susceptibility trends to frequently prescribed medication used for management, minimal evidence is available about the severity of SSIs in Ethiopia. This disparity makes it more difficult for physicians to choose empirical therapy. So, in light of the above facts, the study was aimed to isolate, cultivate, and identify the bacteria from the surgical sites and to assess their susceptibility pattern for the commonly prescribed antibiotics in the study setting.

\section{Methods}

A prospective study was planned and conducted in a 321 bedded hospital, a public sector referral and teaching hospital in Ethiopia. The target departments for the study were general surgical and orthopaedic units. All the 643 individuals who underwent elective surgical interventions between March 2016 and May 2017 were assessed for surgical site infections, and the patients who were suspected of postoperative wound infections were enrolled in the study. The elective surgical procedures/ interventions included laparotomy, appendectomy, prostatectomy, thyroidectomy, debridement, and incisions. The commonly performed surgeries under emergency conditions were exploratory laparotomy and appendectomy.

On the first day of patients' admission to the hospital, the relevant information from the patient was collected by the surgeon. Besides, the surgical site was evaluated by a surgeon (who himself is one of the other operating surgeons) daily during the tenure of the hospital stay, two times a week up to 14 days postoperatively. However, for the observation of other complications, the surveillance time was extended up to 30 days after hospital discharge.

Two experienced clinical pharmacists used pretested structured questionnaires to obtain information from the patient's case files. The information included were demographics (such as age, sex), existing chronic conditions (such as heart disease, metabolic syndrome), and previous medical condition (past diagnosis and cure like cataract surgery, dental restorations, and burn excision). Also, information like current medication, alcoholism, duration of preoperative stay in the hospital, duration of surgery, and, if given, antimicrobial prophylaxis and drain used were gathered using pretested structured questionnaires [11]. In the case of a suspected patient of wound infection, the wound site samples were collected as per the centers' guidelines for disease control and prevention (CDC) [12]. The samples from the wound secretions were collected aseptically by the treating surgeons with the help of sterile cotton swabs without contaminating them with skin floras. All samples were received before cleaning the wound with antiseptic as well as before starting the antibiotic therapy for healing the wound.

Collected swab specimens were sent to the Asella Referral and Teaching Hospital's microbiology laboratory for culture and sensitivity testing to identify, isolate, and understand the isolates' susceptibility pattern against various antibiotics. Standard microbiological procedures like Gram staining, biochemical evaluation, and morphological examinations were applied for the bacterial isolation and identification and drug susceptibility evaluation. According to the standard guidelines, classical biochemical tests were performed for the species identification of the isolates from pure colonies [13]. The antibiotics (drugs) tested for susceptibility for both gram-negative and gram-positive bacteria were ampicillin $(10 \mu \mathrm{g})$, ciprofloxacin $(5 \mu \mathrm{g})$, gentamicin $(10 \mu \mathrm{g})$, chloramphenicol $(30 \mu \mathrm{g})$, doxycycline $(30 \mu \mathrm{g})$, ceftazidime, and ceftriaxone 
$(30 \mu \mathrm{g})$. Methicillin $(5 \mu \mathrm{g})$ and vancomycin $(30 \mu \mathrm{g})$ were used for only gram-positive bacterial isolates. Preanalytical, analytical, and post-analytical stages of quality assurance incorporated in standard operating procedures (SOPs) of the Asella Referral and Teaching Hospital's microbiology laboratory were strictly followed.

\section{Ethical consideration}

The Institutional Ethical Review Committee approved the study (Approval Reference Number: A/CHS/RC005/ 15/16). Written informed consent from each study participant was obtained. All the data collected from participants in the study was kept confidential. The study participant's laboratory outcome was reported to the treating physicians for adequate care.

\section{Statistical analysis}

All data were evaluated statistically (Graph Pad Prism version 5.01), and mean \pm SD was calculated for age. The comparison between the two groups was made by applying a chi-squared test. Significant differences were observed for the two groups based on age, sex, previous antibiotic exposure within 1 month, received antibiotic prophylaxis, and type of antibiotics prescribed for prophylaxis. All comparisons were made at $P \leq 0.05$.

\section{Results}

A total of 643 patients of aged range 23-81 years (mean $\pm \mathrm{SD}=42.4 \pm 13$ years) were examined during the study. Among the 643 evaluated patients, $150 \mathrm{pa}$ tients $(23.3 \%)$ were suspected and positively diagnosed with various kinds of surgical site wound infection, as shown in Table 1. The significant factors for SSI development were age, previous antibiotic exposure, and type of exposure, while sex and antibiotic prophylaxis were not significantly associated with the development of differences, as shown in Table 2.

The surgical procedures of 150 postoperative surgical site infections were observed from patients who had laparotomy $63(42 \%, n=150)$, appendectomy $42(28 \%, n=$ $150)$, prostatectomy $12(8 \%, n=150)$, thyroidectomy 8 $(5.3 \%, n=150)$, and others $25(16.7 \%, n=150)$. The majority of the wound swabs, $122(81.3 \%, n=150)$, developed bacterial growth within 18-24 h of incubation. A mixed growth pattern was observed in 41 patients out of 122 (33.6\%, $n=122)$, while a pure single bacterial growth was observed in 81 patients $(66.4 \%, n=122)$. The rest, 28 patients $(18.7 \%, n=150)$, did not observe any kind of

Table 1 Overall rate of SSI

\begin{tabular}{ll}
\hline Variables & No. of patients (\%) \\
\hline SSI & $150(23.3)$ \\
Without SSI & $493(76.7)$ \\
\hline
\end{tabular}

bacterial growth even after incubation of $48 \mathrm{~h}$ and considered as the uninfected wound.

A total of 147 bacterial isolates were recovered in 122 patients where gram-positive bacteria were $44(29.9 \%$, $n=147)$ and gram-negative bacteria were $103(70.1 \%, n=$ 147) (Table 3). Various bacterial pathogens with SSI, isolated from certain patients were Staphylococcus aureus, coagulase-negative Staphylococcus (CoNS), Enterococcus species, Klebsiella species, Escherichia coli, Pseudomonas aeruginosa, Enterobacter species, Citrobacter species, and Proteus species, were summarized (Table 4). The antimicrobial susceptibility patterns of gram-negative and gram-positive bacterial isolates are presented in Tables 5 and 6.

As shown in Table 4, Klebsiella spp. was considered as the first predominant gram-negative isolate, revealed a higher resistance level to ceftazidime and doxycycline, each 34 (89.4\%, $n=38)$; ampicillin 33 (86.8\%, $n=38$ ); chloramphenicol $31(81.5 \%, n=38)$; and gentamicin and ceftriaxone, each 27 ( $71 \%, n=38)$; and it was found to be sensitive to ciprofloxacin $25(65.8 \%, n=38)$.

According to Table 4, the second predominant gramnegative isolate was $E$. coli, also exhibited the resistance against ampicillin and chloramphenicol, each $26(83.8 \%$, $n=31)$; doxycycline $25(80.6 \%, n=31)$; and gentamicin and ceftazidime, each $16(51.6 \%, n=31)$. It was sensitive to ciprofloxacin $22(71 \%, n=31)$. The first predominant gram-positive isolate, $S$. aureus, displayed some level of resistance to ceftazidime $17(68 \%, n=25)$; doxycycline and gentamicin, each 13 (52\%, $n=25)$; chloramphenicol $12(48 \%, n=25)$; ampicillin $11(44 \%, n=25)$; ciprofloxacin and methicillin, each $9(36 \%, n=25)$; and ceftriaxone 7 $(28 \%, n=25)$, but all the $S$. aureus isolates exhibited sensitivity against vancomycin $25(100 \%, n=25)$.

\section{Discussion}

Nosocomial infections become prominent in surgical wards because of surgical intervention and operative procedures. A total of 147 isolates were recovered from 122 patients in the present study over one and a half years.

In this study, the prevalence of SSI was $23.3 \%$, in contrast to previous studies in Addis Ababa 39\% [14], Mekelle Hospital 44\% [10] in Ethiopia, and 7.3\% in Pakistan [15]. The difference in surgical site infection's magnitude may be due to the type of procedures, surgical setup, and environmental factors. Bacterial isolates were examined for the determination of its categories and antibacterial sensitivity patterns. The present analysis shows that gram-negative bacterial isolates predominate in SSIs; Klebsiella species (26\%) is perhaps the most common isolated pathogen followed by Escherichia coli (21\%), S. aureus (17\%), P. aeruginosa (12\%), CoNS (10\%), Proteus species (4\%), and Enterobacter species 
Table 2 Various factors selected for the study of SSI

\begin{tabular}{|c|c|c|c|}
\hline Variable & $\begin{array}{l}\text { No SSI } \\
N(\%), n\end{array}$ & $\begin{array}{l}\text { SSI } \\
N(\%), n\end{array}$ & $P$ value ${ }^{*}$ \\
\hline \multicolumn{4}{|l|}{ Age } \\
\hline$<50$ years & $297(60.2), n=493$ & $66(44), n=150$ & \multirow[t]{2}{*}{0.0004} \\
\hline$\geq 50$ years & $196(39.8), n=493$ & $84(56), n=150$ & \\
\hline \multicolumn{4}{|l|}{ Sex } \\
\hline Male & $267(54.2), n=493$ & 91 (60.7), $n=150$ & \multirow[t]{2}{*}{0.1600} \\
\hline Female & $226(45.8), n=493$ & 59 (39.3), $n=150$ & \\
\hline \multicolumn{4}{|l|}{ Previous antibiotic exposure } \\
\hline Yes & $325(65.9), n=493$ & $84(56), n=150$ & \multirow[t]{2}{*}{0.0270} \\
\hline No & $168(34.1), n=493$ & $66(44), n=150$ & \\
\hline \multicolumn{4}{|l|}{ Received antibiotic prophylaxis } \\
\hline Yes & $405(82.2), n=493$ & $129(86), n=150$ & \multirow[t]{2}{*}{0.2712} \\
\hline No & $88(17.8), n=493$ & $21(14), n=150$ & \\
\hline \multicolumn{4}{|l|}{ Type of antibiotics prescribed } \\
\hline Ceftriaxone & $219(54.1), n=405$ & $88(68.2), n=129$ & \multirow{3}{*}{0.0178} \\
\hline Ceftriaxone + metronidazole & $170(42), n=405$ & $37(28.7), n=129$ & \\
\hline Ceftriaxone + gentamicin & $16(3.9), n=405$ & $4(3.1), n=129$ & \\
\hline
\end{tabular}

${ }^{*} P$ value $\leq 0.05$ was considered statistically significant

(4\%). The current findings of the SSI infection-causing pathogenic pattern are different with earlier research in Bahir Dar [8] and Gondar [9, 16], where S. aureus is recorded as the widespread SSI-causing organism [17-19]. The reason for the predominancy of gram-negative bacteria might be because most of the infected patients are associated with abdominal surgery, and it is previously reported that gram-negative bacteria are predominantly related to intraabdominal procedures [20]. Findings of this research also indicated that more than $66 \%$ of gramnegative rods were resistant to ampicillin. A previous study also indicated that, in Gondar, Ethiopia, ciprofloxacin was effective for more than $90 \%$ of gram-negative isolates [9]. However, in the present study, ciprofloxacin was effective for more than $61 \%$ of the isolates. This sharp fall in effectiveness may be due to overuse of it as an empiric treatment option for most patients.
Among gram-negative isolates, Klebsiella species, Pseudomonas aeruginosa, and Escherichia coli demonstrated high resistance to most of the antibiotics tested. Eighty-four percent of Klebsiella species, 77\% of Pseudomonas aeruginosa, and 61\% of Escherichia coli were resistant to multiple antibiotics. Although they are not dependable for empiric treatment, ciprofloxacin and ceftriaxone were relatively useful for most bacterial isolates. This finding was also in agreement with the findings of other studies from Bahir Dar and Gondar, Ethiopia [8, 9, 16].

Resistance towards gentamicin in S. aureus differs globally; in our analysis, moderate resistance $(52 \%)$ was observed that was greater than just that recorded at the university hospital in Gondar [16]. A higher incidence of methicillin resistance in $S$. aureus was also recorded in the present study; $36 \%$ of $S$. aureus isolates were

Table 3 Various surgical procedures and corresponding bacterial isolates from patients

\begin{tabular}{lllll}
\hline $\begin{array}{l}\text { Site of surgical } \\
\text { procedures }\end{array}$ & $\begin{array}{l}\text { Patients } \\
\text { No. (\%) }\end{array}$ & Bacterial isolates & \\
\cline { 3 - 5 } & Gram-positive No. (\%) & Gram-negative No. (\%) & Total isolates No. \\
\hline Laparotomy & $53(43.2)$ & $15(19.7)$ & $61(80.3)$ & 76 \\
Appendectomy & $32(26.2)$ & $21(41.2)$ & $30(58.8)$ & 51 \\
Prostatectomy & $12(9.8)$ & $2(14.4)$ & $11(84.6)$ & 13 \\
Thyroidectomy & $8(6.6)$ & $2(66.7)$ & $1(33.3)$ & 3 \\
Debridement & $11(9)$ & $3(100)$ & 0 & 3 \\
Incision & $6(4.9)$ & $1(100)$ & 0 & 1 \\
Total & 122 & $44(29.9)$ & $103(70.1)$ & 147 \\
\hline
\end{tabular}


Table 4 Bacterial pathogens from patients with SSI

\begin{tabular}{|c|c|c|}
\hline \multicolumn{2}{|l|}{ Bacterial isolates } & \multirow{2}{*}{$\frac{\text { No. (\%), } \boldsymbol{n}}{25(56.8), n=44}$} \\
\hline Gram-positive isolates $44(29.9 \%, n=147)$ & Staphylococcus aureus & \\
\hline & CoNS & $15(34.1), n=44$ \\
\hline & Enterococcus species & $4(9.1), n=44$ \\
\hline \multirow[t]{6}{*}{ Gram-negative isolates $103(70.1 \%, n=147)$} & Klebsiella species & $38(36.9), n=103$ \\
\hline & Escherichia coli & $31(30.1), n=103$ \\
\hline & Pseudomonas aeruginosa & $18(17.5), n=103$ \\
\hline & Proteus species & $6(5.8), n=103$ \\
\hline & Enterobacter species & $5(4.9), n=103$ \\
\hline & Citrobacter species & $5(4.9), n=103$ \\
\hline
\end{tabular}

methicillin-resistant Staphylococcus aureus (MRSA). The finding was higher than the recent report from Gondar University Hospital within the country (24\%) [16], and in Mwanza, Tanzania (18.8\%) [18].

In our experiment, gram-negative microbes exhibited higher resistance to widely recommended cost-effective antibiotics like penicillin derivatives, doxycycline, and chloramphenicol. The observed high resistance rate could be attributed because these lowpriced antimicrobial agents are simple to administer, relatively inexpensive, and commonly prescribed in the evidential management of different infectious diseases. In order to efficiently treat surgical site infections, the use of such medications must be strictly examined for the therapeutic outcome and must be driven by microbiological tests. In the present study, resistance to the third-generation cephalosporins in gram-negative pathogens was higher, and these findings are similar to many prior reports from the Bahir Dar and Gondar regions of Ethiopia, and other nations $[9,16,21]$. An intensified unnecessary prescribing of ceftriaxone as a surgical prophylactic antibiotic at this referral hospital may be a potential cause for the observed greater resistance. Research on antibiotic usage in the same hospital reported ceftriaxone as the most frequently used injectable medication, with some more than 300 prescriptions each month in the surgical ward itself, as a piece of unpublished information from the hospital drugstore.

Besides, several patients from this study got ceftriaxone as a prophylactic antibiotic to avoid infections at the surgical site, and that may have disrupted the exposure of gram-negative pathogens susceptible to ceftriaxone. Most of the Pseudomonas aeruginosa strains isolated were highly sensitive to ceftazidime and moderately to ciprofloxacin and gentamicin. These findings agree with those studies from Gondar University Hospital $[9,16]$ and Karachi, Pakistan [15]. The above information demonstrates the ability of these classes of medicines for prescribing to treat Pseudomonas in SSIs.

\section{Limitation of the study}

Due to the limited resources, individual characterization of the isolates at the species level like Klebsiella spp., Proteus spp., Enterobacter spp., CoNS, Citrobacter spp., Enterobacter spp. was not possible to be performed.

\section{Conclusions}

The SSI rate was found to be $23.3 \%$. The predominant isolates were Klebsiella spp, E. coli, S. aureus, P. aeruginosa, ConS, Proteus spp, and Enterobacter spp. The findings of research explored that gram-negative wound pathogens exhibited a significantly higher isolation rate $(P<0.5)$ than gram-positive pathogens, with high

Table 5 Antimicrobial susceptibility patterns of gram-negative bacterial isolates from wound swab cultures of postoperative surgical site infections

\begin{tabular}{|c|c|c|c|c|c|c|c|}
\hline \multirow[t]{2}{*}{ Bacterial isolates } & \multicolumn{7}{|c|}{ Number of resistant pathogens to antimicrobial agents (\%) } \\
\hline & $\overline{\mathrm{GN}}$ & CIP & CEFTA & AMP & CEF & CAF & DOX \\
\hline Klebsiella spp. $(n=38)$ & $27(71)$ & $13(34.2)$ & $34(89.4)$ & $33(86.8)$ & $27(71)$ & $31(81.5)$ & $34(89.4)$ \\
\hline Escherichia coli $(n=31)$ & $16(51.6)$ & $9(29)$ & $16(51.6)$ & $26(83.8)$ & $6(19.3)$ & $26(83.8)$ & $25(80.6)$ \\
\hline Pseudomonas aeruginosa $(n=18)$ & $11(61.1)$ & $10(55.5)$ & $2(11.1)$ & $16(88.8)$ & $15(83.3)$ & $16(88.8)$ & $16(88.8$ \\
\hline Proteus spp. $(n=6)$ & $1(16.6)$ & $5(83.3)$ & $1(16.6)$ & $5(83.3)$ & $3(50)$ & $1(16.6)$ & $2(33.3)$ \\
\hline Enterobacter spp. $(n=5)$ & $1(20)$ & $1(20)$ & $2(40)$ & $4(80)$ & $2(40)$ & $4(80)$ & $2(40)$ \\
\hline Citrobacter spp. $(n=5)$ & $4(80)$ & $2(40)$ & $4(80)$ & $4(80)$ & $4(80)$ & $4(80)$ & $4(80)$ \\
\hline
\end{tabular}

GN gentamicin, CIP ciprofloxacin, CEFTA ceftazidime, AMP ampicillin, CEF ceftriaxone, CAF chloramphenicol, DOX doxycycline 
Table 6 Antimicrobial susceptibility patterns of gram-positive bacterial isolates from wound swab cultures of postoperative surgical site infections

\begin{tabular}{llllllllll}
\hline Bacterial isolates & \multicolumn{9}{l}{ Number of resistant pathogens to antimicrobial agents (\%) } \\
\cline { 2 - 9 } & GN & CIP & CEFTA & AMP & CEF & VAN & CAF & MET & DOX \\
\hline S. aureus $(n=25)$ & $13(52)$ & $9(36)$ & $17(68)$ & $11(44)$ & $7(28)$ & $0(0)$ & $12(48)$ & $9(36)$ & $13(52)$ \\
CoNS $(n=15)$ & $7(46.6)$ & $3(20)$ & $7(46.6)$ & $5(33.3)$ & $3(20)$ & $0(0)$ & $8(53.3)$ & $5(33.3)$ & $8(53.3)$ \\
Enterococcus spp. $(n=4)$ & $0(0)$ & $0(0)$ & $0(0)$ & $0(0)$ & $0(0)$ & $0(0)$ & $0(0)$ & $1(25)$ & $0(0)$ \\
\hline
\end{tabular}

GN gentamicin, CIP ciprofloxacin, CEFTA ceftazidime, AMP ampicillin, CEF ceftriaxone, VAN vancomycin, CAF chloramphenicol, Met methicillin, DOX doxycycline, CoNS coagulase-negative Staphylococcus

resistance rates. Most gram-negative wound isolates were found to be resistant against commonly advised antibiotics. On the other hand, most isolated gram-positive pathogens were sensitive towards vancomycin, except one Enterococcus species. To achieve effective therapy for wound infections and to reduce/stop the appearance of multidrug-resistant (MDR) pathogens, continuous monitoring is essential with fair use of antimicrobial agents.

\section{Abbreviations}

SSI: Surgical site infection; S. aureus: Staphylococcus aureus; E. coli: Escherichia coli; P. aeruginosa: Pseudomonas aeruginosa; CDC: Centers for Disease Control and Prevention; spp: Species; SOP: Standard operating procedure;

SD: Standard deviation; CoNS: Coagulase-negative Staphylococcus;

GN: Gentamicin; CIP: Ciprofloxacin; CEFTA: Ceftazidime; AMP: Ampicillin;

CEF: Ceftriaxone; VAN: Vancomycin; CAF: Chloramphenicol; Met: Methicillin;

DOX: Doxycycline; MRSA: Methicillin-resistant Staphylococcus aureus;

MDR: Multidrug resistant

\section{Acknowledgements}

The authors would like to acknowledge the Arsi University Research and Publication Office for funding this project. The authors also thank the study participants, research and community service coordinators of the college of health sciences, Arsi University, and the microbiology laboratory technologists for collecting the sample and using the laboratory services. The authors are very thankful to the Asella Referral and Teaching Hospital surgical ward staffs for their cooperation during the data collection.

\section{Authors' contributions}

TG conceived and designed the research idea and performed proposal writing, data collection, laboratory work, and analysis. BB participated in the study design, analysis, and interpretations of the findings. MY participated in data analysis and interpretations, and write-up. SM participated in the study design, data collection, and laboratory work, and KMN participated in the data analysis and interpretations of the findings, write-up, manuscript writing, and reviewing. All authors were involved in reviewing the manuscript and approval for publication.

\section{Funding}

Arsi University Research and Publication Office, Asella, Ethiopia, financially supported our study. The funders had no role in study design, data collection and analysis, decision to publish, or manuscript preparation.

\section{Availability of data and materials}

The datasets used and analyzed during the study are available on reasonable request.

\section{Declarations}

\section{Ethics approval and consent to participate}

Ethical approval was obtained from the College of Health Sciences Arsi University Ethical Review Committee (CHSAUERC), Arsi University, Asella, Ethiopia (approval reference number: A/CHS/RC005/15/16). The study was carried out at Asella Referral and Teaching Hospital, a public sector referral and teaching hospital affiliated to Arsi University College of Health Sciences. Written informed consent was obtained from each study participant. All the data collected from participant in the study was kept confidential.

\section{Consent for publication}

A consent for publication was obtained from all the participants while obtaining the consent to participate.

\section{Competing interests}

The authors declare that they have no competing interests.

\section{Author details}

${ }^{1}$ Department of Pharmacy, College of Health Sciences, Arsi University, Asella, Oromia, Ethiopia. ${ }^{2}$ Department of Surgery, College of Health Sciences, Arsi University, Asella, Oromia, Ethiopia.

Received: 2 January 2021 Accepted: 28 April 2021

Published online: 08 May 2021

\section{References}

1. Rhomberg PR, Fritsche TR, Sader HS, Jones RN (2006) Antimicrobial susceptibility pattern comparisons among intensive care unit and general ward gram-negative isolates from the Meropenem Yearly Susceptibility Test Information Collection Program (USA). Diagn Microbiol Infect Dis 56(1):5762. https://doi.org/10.1016/j.diagmicrobio.2005.12.009

2. Weigelt JA, Lipsky BA, Tabak YP, Derby KG, Kim M, Gupta V (2010) Surgical site infections: causative pathogens and associated outcomes. Am J Infect Control 38(2):112-120. https://doi.org/10.1016/j.ajic.2009.06.010

3. Tacconelli E, De Angelis G, Cataldo MA, Mantengoli E, Spanu T, Pan A, Corti G, Radice A, Stolzuoli L, Antinori S, Paradisi F, Carosi G, Bernabei R, Antonelli M, Fadda G, Rossolini GM, Cauda R (2009) Antibiotic usage and risk of colonization and infection with antibiotic-resistant bacteria: a hospital population-based study. Antimicrob Agents Chemother 53(10):4264-4269. https://doi.org/10.1128/AAC.00431-09

4. Bibi S, Channa GA, Siddiqui TR, Ahmed W (2012) Pattern of bacterial pathogens in postoperative wounds and their sensitivity patterns. J Surg Pak (Int) 17(4):164-167

5. Al-Momany NH, Al-Bakri AG, Makahleh ZM, Wazaify MM (2009) Adherence to international antimicrobial prophylaxis guidelines in cardiac surgery: a Jordanian study demonstrates need for quality improvement. J Manag Care Pharm 15(3):262-271. https://doi.org/10.18553/jmcp.2009.15.3.262

6. Kotisso B, Aseffa A (1998) Surgical wound infection in a teaching hospital in Ethiopia. East Afr Med J 75(7):402-405

7. Tietjen L, Bossemeyer D, McIntosh N (2003) Infection prevention guidelines for healthcare facilities with limited resources. JHPIEGO Corporation, Baltimore, p 23-3

8. Fantahun B, Beyeh A, Atenaf A, Belay A (2009) Bacterial isolates from wound infection and their antimicrobial susceptibility pattern in Felege Hiwot referral hospital, Northwest Ethiopia. Ethiop J Health Sci 19(3):173-177

9. Mulu A, Moges F, Tessema B, Kassu A (2006) Pattern and multiple drug resistance of bacterial pathogens isolated from wound infection at University of Gondar Teaching Hospital, Northwest Ethiopia. Ethiop Med J 44(2):125-131

10. Tesfahunegn Z, Asrat D, Woldeamanuel Y, Estifanos K (2009) Bacteriology of surgical site and catheter related urinary tract infections among patients admitted in Mekelle Hospital, Mekelle, Tigray, Ethiopia. Ethiop Med J 47(2): $117-127$ 
11. Horan TC, Gaynes RP, Martone WJ, Jarvis WR, Emori TG (1992) CDC definitions of nosocomial surgical site infections, 1992: a modification of CDC definitions of surgical wound infections. Infect Control Hosp Epidemiol 13(10):606-608. https://doi.org/10.2307/30148464

12. Sangrasi AK, Leghari AA, Memon A, Talpur AK, Qureshi GA, Memon JM (2008) Surgical site infection rate and associated risk factors in elective general surgery at a public sector medical university in Pakistan. Int Wound J 5(1):74-78. https://doi.org/10.1111/j.1742-481X.2007.00365.x

13. College of Physicians \& Surgeons of Saskatchewan Laboratory Quality Assurance Program (2010) Procedures/guidelines for the microbiology laboratory. College of Physicians \& Surgeons of Saskatchewan Laboratory Quality Assurance Program, Saskatchewan

14. Endalafer N, Gebre-Selassie S, Kotiso B (2011) Nosocomial bacteria infections in a tertiary hospital in Ethiopia. J Infect Prev 12(1):38-43. https:// doi.org/10.1177/1757177410376680

15. Mahmood A (2000) Bacteriology of surgical site infections and antibiotic susceptibility pattern of the isolates at a tertiary care hospital in Karachi. J Pak Med Assoc 50(8):256-259

16. Aschalew G, Solomon GS, Moges T, Eshetu M, Sisay Y (2014) Isolation of bacterial pathogens from patients with postoperative surgical site infections and possible sources of infections at University of Gondar Hospital, Northwest Ethiopia. J Environ Occup Health 3(2):103-108. https://doi.org/10. 5455/jeos.20140512124135

17. Andhoga J, Macharia AG, Maikuma IR, Wanyonyi ZS, Ayumba BR, Kakai R (2002) Aerobic pathogenic bacteria in postoperative wounds at Moi Teaching and Referral Hospital. East Afr Med J 79(12):640-644. https://doi. org/10.4314/eamj.v79i12.8671

18. Mawalla B, Mshana SE, Chalya PL, Imirzalioglu C, Mahalu W (2011) Predictors of surgical site infections among patients undergoing major surgery at Bugando Medical Centre in Northwestern Tanzania. BMC Surg 11:21. https:// doi.org/10.1186/1471-2482-11-21

19. Como-Sabetti KJ, Harriman KH, Fridkin SK, Jawahir SL, Lynfield R (2010) Risk factors for community-associated Staphylococcus aureus infections: results from parallel studies including methicillin-resistant and methicillin-sensitive S. aureus compared to uninfected controls. Epidemiol Infect 139(3):419-429. https://doi.org/10.1017/S0950268810001111

20. Koigi-Kamau R, Kabare LW, Wanyoike-Gichuhi J (2005) Incidence of wound infection after caesarean delivery in a district hospital in central Kenya. East Afr Med J 82(7):357-361

21. Anguzu JR, Olila D (2007) Drug sensitivity patterns of bacterial isolates from septic postoperative wounds in a regional referral hospital in Uganda. Afr Health Sci 7(3):148-154. https://doi.org/10.5555/afhs.2007.7.3.148

\section{Publisher's Note}

Springer Nature remains neutral with regard to jurisdictional claims in published maps and institutional affiliations.

\section{Submit your manuscript to a SpringerOpen ${ }^{\circ}$ journal and benefit from:}

- Convenient online submission

- Rigorous peer review

- Open access: articles freely available online

- High visibility within the field

- Retaining the copyright to your article

Submit your next manuscript at $\boldsymbol{\nabla}$ springeropen.com 